\title{
Spinal granulocytic sarcoma (chloroma) presenting as acute cord compression in a nonleukemic patient
}

\author{
Murat Kalayci, Murat Sümer', Sibel Yenidünya², Senay Özdolap³ ${ }^{2}$ Bektas Açikgöz \\ Departments of Neurosurgery, ${ }^{1}$ Neurology, ${ }^{2}$ Pathology and ${ }^{3}$ Physical Medicine and Rehabilitation, Zonguldak Karaelmas University Medical \\ School, Kozlu, Zonguldak, Turkey
}

\begin{abstract}
The case of a previously healthy 24 -year-old man diagnosed with extradural thoracic granulocytic sarcoma with no evidence of bone marrow or other hematological involvement is described. The tumor was removed totally by microsurgery. The histopathological examination was consistent with granulocytic sarcoma. Granulocytic sarcomas are most commonly found in the context of an acute myelogenous leukemia or in chronic myelogenous leukemia. They rarely have been reported in otherwise healthy patients without any evidence of systemic disease. A review of the literature revealed only 14 more nonleukaemic cases with granulocytic sarcoma causing thoracic spinal cord compression.
\end{abstract}

Key Words: Chloroma, epidural mass, granulocytic sarcoma, spine

Granulocytic sarcomas (chloroma) originate from malignant myeloid blast cells and infiltrate the spinal extradural area. ${ }^{[1]-}$ [4] They are rare manifestations of acute and chronic leukemia and can occasionally precede the development of systemic disease by weeks to years. ${ }^{[1],[2],[4],[5]}$ Granulocytic sarcomas may occur in the extramedullary spinal canal and cause spinal cord compression or cauda equina syndrome. Only a few cases of granulocytic sarcoma presenting with symptoms of spinal cord compression in patients without myelogenous leukemia have been reported. ${ }^{[2],[4]-[11]}$

We discuss a patient with primary granulocytic sarcoma of the thoracic spine and present a brief literature on the subject.

\section{Case report}

A 24-year-old man had localized back pain with bilateral lower extremity weakness, numbness, and urinary retention for about 45 days. His medical history was otherwise unremarkable. Physical ex- amination revealed no abnormality. Sensory assessment revealed hypoesthesia below T5 and motor examination revealed a 3/5 paraparesis. He was unable to stand or walk independently. Deep tendon reflexes were hyperactive and Babinski signs were bilaterally positive. Laboratory evaluation revealed a white blood cell count of 4.100/ $\mathrm{mm}^{3}$, a hemoglobin level of $12.6 \mathrm{~g} / \mathrm{dl}$, and an hematocrit of $37.2 \%$. Magnetic resonance imaging (MRI) of the thoracolumbar spine revealed a posterior epidural mass between T3 and T5 [Figure 1A].

High-dose ( $8 \mathrm{~g} /$ day) methylprednisolone was given initially, and he underwent emergent spinal cord decompression with T3 and T4 laminectomy. A soft and grayish tumor compressing the spinal cord was identified. Gross total tumor resection was carried out [Figure 1B]. Postoperatively, the patient underwent a bone marrow biopsy, which revealed normocellular bone marrow with no evidence of lymphoma or leukemia. Peripheral blood examination was normal.

Histologically the tumor was composed of a relatively uniform population of immature cells [Figure 2]. Cells with bright eosinophilic, occasionally granular cytoplasm, consistent with myeloid lineage were present. The myeloblast had a slight to moderate rim of basophilic cytoplasm, fine nuclear chromatin, and two to four nucleoli. Eosi-
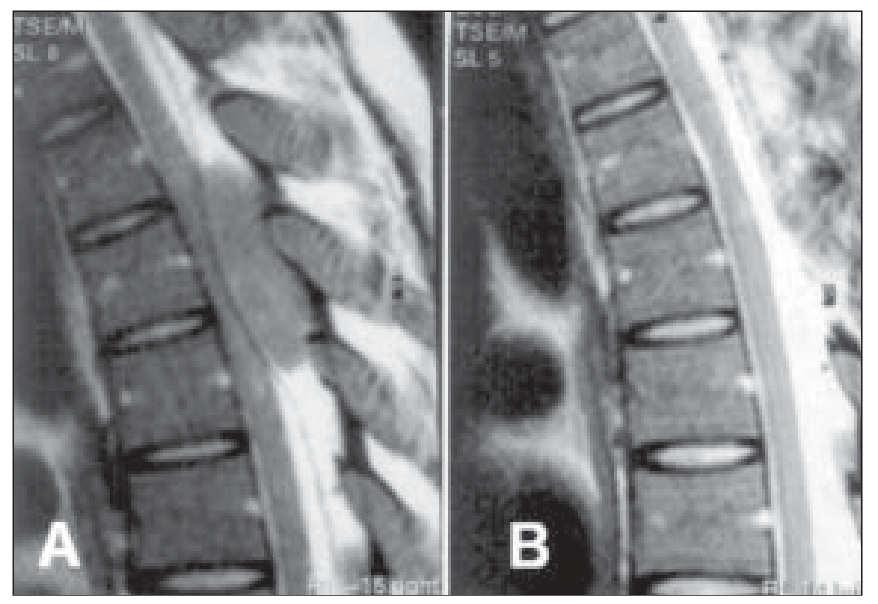

Figure 1: (A) Sagittal T2-weighted magnetic resonance imaging (MRI) scans demonstrating the large epidural mass from T3 to T5. (B) Postoperative sagittal T2-weighted MRI scans demonstrating adequate spinal cord decompression 


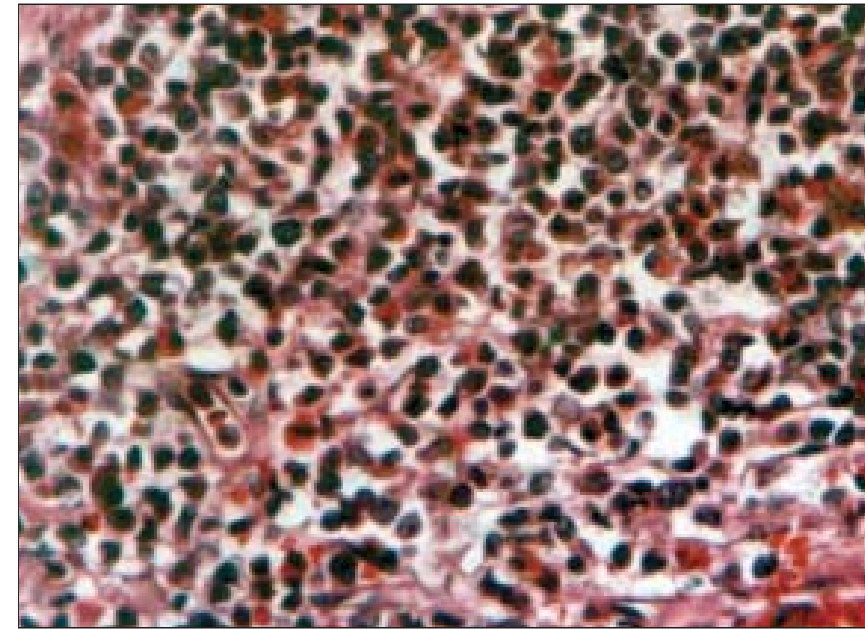

Figure 2: Biopsy of epidural granulocytic sarcoma. Numerous blasts are present. H\&E (original magnification, $x 40$ )

nophil myelocytes were abundant. Because of a prominent component of myelocyte, with round, eccentric nuclei, and moderately abundant cytoplasm, and because of an associated chronic inflammatory cell infiltrate that contained mature plasma cells, the mass was initially misinterpreted as plasmacytoma. Because of the presence of a uniform population of primitive cells with scant cytoplasm, malignant lymphoma was also suspected. The presence of immature eosinophils and maturing neutrophils indicated the true nature of the tumor. Immunohistochemical staining revealed the expression of myeloperoxidase, lysozyme, and leukocyte common antigen and not of B-cell-specific or T-cell-specific antigens [Figure 3]. The final pathological diagnosis of the specimen was granulocytic sarcoma.

Although bone marrow biopsy results were inconsistent with leukemia, we preferred antileukemic therapy with a standard chemotherapeutic protocol and radiation therapy to the spinal axis and the tumor bed, because of the possibility of late development of leukemia. With an aggressive physical therapy regimen, he gradually gained strength in his lower extremities and regained the ability to walk with a cane. Bladder function was normal. No evidence of acute myelogenous leukemia has emerged over 8 months of follow up.

\section{Discussion}

Granulocytic sarcomas represent a diagnostic challenge, particularly those occurring in patients without evidence of systemic disease. ${ }^{[2],[6],[11]}$ Granulocytic sarcomas have been associated with 3.1-9.1\% of acute myelogenous leukemia cases. ${ }^{[2]}$ Rarely, they can occur alone without peripheral blood or bone marrow evidence of leukemia. ${ }^{[5]}$ Granulocytic sarcomas are often the initial presenting feature of acute myelogenous leukemia, even when both are present at diagnosis. ${ }^{[2],[4],[6]}$ Late development of acute myelogenous leukemia frequently occurs. The interval between initial diagnosis and the onset of acute leukemia has been reported between 1 and 49 months (average: 10 months). ${ }^{[2]}$ We could find only 14 patients with primary epidural granulocytic sarcoma of the spine in the literature. $^{[2],[4]-[11]}$

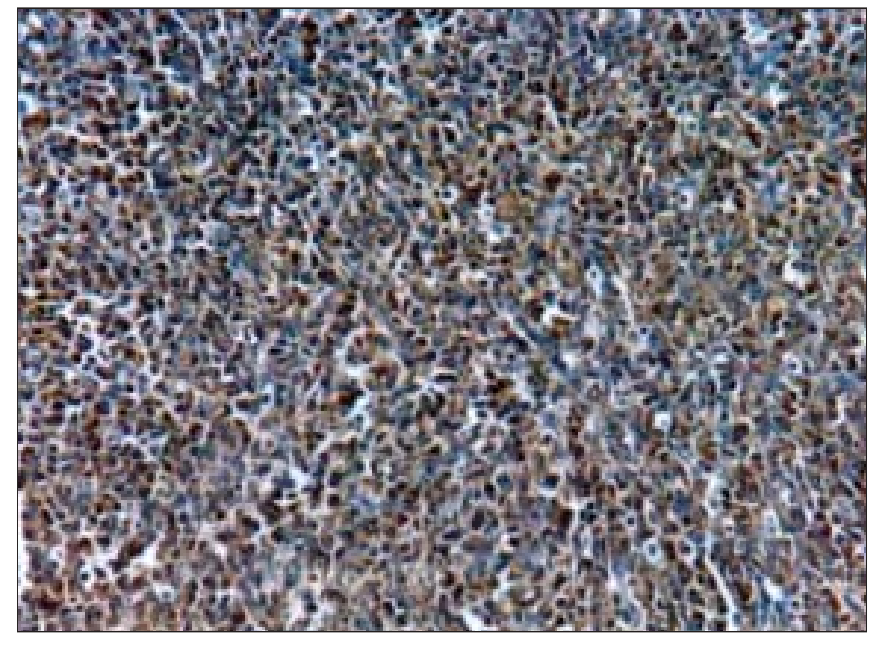

Figure 3: Same specimen reacted with antibody to myeloperoxidase. Virtually all blasts are positive (original magnification, x20)

Granulocytic sarcomas usually occur in the bone, skin, and lymph nodes. Bony involvement is most common in the orbit, sacrum, spine, and ribs. ${ }^{[3],[5],[6]}$ The origin of the tumor is thought to be the bone marrow, with migration to extraosseous locations achieved via the Haversian canals. ${ }^{[2]}$ Macroscopically, granulocytic sarcoma is usually green in appearance. The green color, which is due to the presence of myeloperoxidase in the leukemia cells, is not present in all tumors of this type. This color rapidly fades when exposed to air. ${ }^{[2],[4],[5]}$

The diagnosis is often difficult in granulocytic sarcoma cases when the myeloblastic cells are poorly differentiated and the tumor lacks the characteristic green color. The most common misdiagnoses included large cell lymphoma, malignant lymphoma, reticulum cell lymphoma, and histiocytic lymphoma. ${ }^{[2],[5]}$ A diagnosis of granulocytic sarcoma is not ruled out when bone marrow biopsy specimens show no evidence of leukemia infiltration. ${ }^{[4]}$

Granulocytic sarcoma should be considered in the differential diagnosis of an epidural mass in patients with or without acute leukemia, because early diagnosis followed by appropriate combined chemotherapy and radiation may obviate surgical intervention and eventually prevent leukemic transformation. ${ }^{[2],[4]-[6]}$ Differential diagnosis includes non-Hodgkin's lymphoma, neuroblastoma, Ewing's sarcoma, rhabdomyosarcoma, and osteosarcoma. ${ }^{[1],[6]}$ The incidence of granulocytic sarcomas is rising, due mostly to the longer survive of acute myelogenous leukemia patients. ${ }^{[2],[6]}$

Treatment options include surgical decompression, chemotherapy, radiation therapy or any combination of these treatment methods. ${ }^{[1],[2],[4],[5]}$ Surgery is generally preferred for cases of acute spinal cord compression. Pathological examination of the obtained tissue from the mass is very important in diagnosis especially in cases without systemic evidence of leukemia. ${ }^{[2],[4],[6]}$ Granulocytic sarcomas are radiosensitive and are often treated by local radiotherapy and systemic chemo- 
therapy. Newly diagnosed patients with isolated granulocytic sarcomas are usually treated with aggressive chemotherapy as if they have acute myelogenous leukemia; cures are not attained with radiation therapy alone. ${ }^{[2]}$

\section{References}

1. Fiegl M, Rieger C, Braness J, Haferlach T, Schnittger S, Schoch C, et al. Isolated epidural chloroma with translocation $\mathrm{t}(15 ; 17)$ successfully treated with chemotherapy and ALL-trans-retinoic acid. Br J Haematol 2003;122:686-91

2. Mostafavi H, Lennarson PJ, Traynalis VC. Granolocytic sarcoma of the spine. Neurosurgery 2000;46:78-84.

3. Suzer T, Colakoglu N, Cirak B, Keskin A, Coskun E, Tahta K. Intracerebellar granulocytic sarcoma complicating acute myelogenous leukemia: A case report and review of the literature. J Clin Neurosei 2004;11:914-7.

4. Ugras S, Cýrak B, Karakok M, Guven B. Spinal epidural granulocytic sarcoma (chloroma) in a non-leukemic child. Pediatr Int 2001;43:505-7.

5. Ripp D.J, Davis .JW, Rengachary SS, Lotuaco LG, Watanabe IS. Granulocytic sarcoma presenting as an epidural mass with cord compression. Neurosurgery
$1989 ; 24: 125-8$.

6. Buckland ME, Scolyer RA, Donellan MB, Brew S, McGee-Collett, Harper CG. Spinal chloroma presenting with triplegia in an aleukaemic patient. Pathology 2001;33:386-9

7. Deme S, Deodhare SS, Tucker WS, Bilbao JM. Granulocytic sarcoma of the spine in nonleukemic patients: Report of three cases. Neurosurgery 1997;40:1283-7.

8. Fiorillo A, de Rosa G, Fariello I, D'Amore R, Bonavolonta G. Granulocytic sarcoma in nonleukemic children: report of two new cases successfully treated by local radiation therapy and systemic chemotherapy. Haematologica 1996;81:155-8

9. Frohna B.J, Quint DJ. Granulocytic sarcoma (chloroma) causing spinal cord compression. Neuroradiology 1993;357:509-11.

10. Kook H, Hwang T.J, Choe K, Yang DW, Nam JH, Park CS. Spinal epidural granulocytic sarcoma preceding acute myelogenous leukemia. J Korean Med Sci 1992; 7:291-6.

11. Kwatra KS, Prabhakar BR, Arora Y. Bilateral granulocytic sareoma (chloroma) of the breast in CML in blast crisis: A case report. Indian J Pathol Vicrobiol 2004:47:66-8

Accepted on 31-12-2004 